\section{Interference with the McCollough effect}

\author{
C. C. D. SHUTE \\ Physiological Laboratory, University of Cambridge \\ Cambridge, England
}

Skowbo, Gentry, Timney, and Morant (1974) found that decay of the McCollough effect (ME) was accelerated by postadaptation exposure to alternate vertical and horizontal achromatic gratings, and later White and Graves (1976) showed that preadaptation exposure to achromatic gratings also produced this effect. Further investigations by Skowbo and Clynes (1977) revealed that the decrease in ME strength due to achromatic gratings was greatest when the inspecting time was long $(30 \mathrm{~min})$, whereas the recovery from such a decrease was greatest with a short inspecting time (10 $\mathrm{min})$. Long inspection of chromatic gratings during adaptation causes an increase in the strength of the resultant ME. Increasing the luminance of the adapting gratings has a similar effect on the ME, and Skowbo (1979) has recently shown that high luminance increases the power of achromatic gratings to interfere with the ME. There seems to be, therefore, some similarity between the conditions that are optimal for setting up the ME and those which favor its suppression. Accordingly, it has been suggested by White and Graves (cited by Skowbo \& Clynes, 1977) and by Skowbo (1979) that interference may be due to an achromatic analogue of the ME biasing the visual system away from chromaticity and towards a neutral state.

Skowbo et al. (1974) did not find evidence of ME suppression by alternating homogeneous colored fields, or by "natural" visual stimulation from objects in the environment, or by complete darkness-from which it might seem that the interference produced by achromatic gratings is in some way specific to them. Further work, however, has shown that a number of diverse factors can influence ME strength. Factors that can interfere with the ME so as to reduce its strength and accelerate its decay include coffee and caffeine (Amure, 1979; Shute, 1978) and also moderate muscular activity and stimulation of a stressful nature (Shute, 1979). Alternating contrasting colors superimposed on the test grating are particularly effective in eradicating the ME (Shute, 1979). Influences having the opposite effect, enhancing the ME or slowing its decay, include extreme fatigue, anticholinergic (acetylcholine blocking) drugs such as hyoscine, and other drugs such as the minor tranquilizers (benzodiazepines) that probably potentiate inhibitory neurotransmitter substances released in the brain (Shute, 1978, 1979).

These findings, pharmacological and others, as well as the observations of Skowbo and co-workers, are most readily interpreted in terms of an inhibitory hypothesis for the ME, according to which the adapting color channel in the visual system is inhibited when the pattern originally associated with that color is re-presented as the test stimulus, and in consequence the opponent color channel becomes secondarily activated. It is suggested that factors which interfere with the ME counter the primary inhibition of the adapting channel by stimulating the cholinergic reticular activating system. The consequent disinhibition results in weaker MEs and faster decay. The actual ME strength at any time can be regarded as resulting from the balance of inhibitory and activating (or excitatory) processes. Shute and Lewis $(1963,1967)$ have demonstrated the existence of acetylcholinesterase-containing, presumed cholinergic nerve terminals at various visual sites, and in some regions of the brain such neurones have, in fact, been proved to be cholinergic. Moreover, acetylcholine has been shown to excite cell firing at visual relays and in visual cortex (Satinsky, 1967; Spehlmann, 1963, 1971). The excitatory cholinergic system is itself activated by collaterals of visual neurones. Naturally, this activation will be particularly effective with strong interfering stimuli such as contrasting gratings with high luminance and long exposure.

An important corollary of this concept is that changes in the normal rate of ME decay can provide an indication of the effectiveness of different stimuli in activating the visual system. This may have particular relevance to the understanding and treatment of amblyopia. It seems likely that in this condition there may be an element of functional inhibition suppressing visual performance in the affected eye. Campbell, Hess, Watson, and Banks (1978) have shown that rotating achromatic gratings can produce rapid clinical improvement in some amblyopic children, especially if the viewing is combined with other attention-catching procedures. It is possible that in refractory cases the stimulatory effect of gratings on the lazy eye might be enhanced by the introduction of additional activating factors, revealed through their effect on the ME.

\section{REFERENCES}

Amure, B. C. Caffeine and the decay of the McCollough effect (ME). Journal of Physiology, London, 1979, Proceedings, 295, 32P.

Campbell, F. W., Hess, R. F., Watson, P. G., \& Banks, R. Preliminary results of a physiologically based treatment of amblyopia. British Journal of Opthalmology, 1978, 62, 748-755.

SAtinsky, D. Pharmacological responsiveness of lateral geniculate nucleus neurones. International Journal of Neuropharmacology, 1967, 6, 387-397. 
Shute, C. C. D. Influence of centrally active drugs on the McCollough effect (ME). Journal of Physiology, London, 1978, 278, 47P.

Shute, C. C. D. The McCollough effect. Cambridge: Cambridge University Press, 1979.

Shute, C. C. D., \& Lewis, P. R. Cholinesterase-containing systems of the brain of the rat. Nature, London, 1963, 199, 1160-1164.

Shute, C. C. D., \& Lewis, P. R. The ascending cholinergic reticular system: Neocortical, olfactory and subcortical projections, Brain, 1967, 90, 447-520.

Skowво, D. Luminance as a factor in the ability of achromatic gratings to interfere with McCollough effects. Perception \& Psychophysics, 1979, 26, 105-107.

Skowbo, D., \& Clynes, N. Decline and revival of McCollough effects following inspection of achromatic gratings, Perception \& Psychophysics, 1977, 21, 180-182.

Skowbo, D., Gentry, T., Timney, B., \& Morant, R. B. The McCollough effect: Influence of several kinds of visual stimulation on decay rate. Perception \& Psychophysics, 1974, 16, 47-49.

Spenlmann, R. Acetylcholine and prostigmine electrophoresis at visual cortex neurones. Journal of Neurophysiology, 1963, 26, 127-139.

Spenlmann, R. Acetylcholine and the synaptic transmission of nonspecific impulses to the visual cortex. Brain, 1971, 94, 139-150.

(Received and accepted for publication October 22, 1979.) 